\title{
On Deformations of Solutions of Involutive Partial Differential Equations
}

\author{
By \\ Kazuhiro Kiso*
}

\section{Introduction}

Let $E, F$ be real analytic vector bundles over a real analytic compact manifold $M$ and $D: \Gamma(E) \rightarrow \Gamma(F)$ a real analytic polynomial differential operator satisfying $D(0)=0$. Let $s(t)$ be a parametrized family of cross sections of $E$, where $t$ moves in some neighborhood of 0 in a euclidean space. We say that $s(t)$ is a deformation of the solution 0 if $s(0)=0$ and $D(s(t))=0$. In the present paper, we will show the existence of deformations of the solution 0 under some conditions. Namely, let $L$ be the linearized differential operator of $D$ at 0 and assume: (1) the equation $D(s)=0$ is involutive, (2) $L$ is elliptic, (3) $H^{1}(M, \Theta)=0$, where $\Theta$ is the solution sheaf of the equation $L(s)=0$. Then we can prove that there is a deformation $s(t)$ which is complete at $t=0$ in an appropriate sense. (Theorem 1,2)

We would like to point out the analogy between the above result and a theorem in [3] on the existence of deformations of complex structures. In fact, the arguments proceed along almost the same line as in [3].

In $\S 1$, we prove some propositions which are needed in the later sections. In $\S 2$, we construct the deformation $s(t)$ and prove its completeness in $\$ 3$.

Finally the author wishes to express his hearty gratitude to Professor N. Tanaka for his constant encouragement and valuable suggestions during the preparation of this paper.

Communicated by S. Nakano, September 3, 1974.

* Graduate School, Kyoto University, Kyoto. 


\section{§1. Differential Equations}

We always assume real analyticity, so that the subscription "real analytic" will be omitted.

Let $\pi: E \rightarrow M, \rho: F \rightarrow M$ be vector bundles over a compact manifold $M$ and $D: \Gamma(E) \rightarrow \Gamma(F)$ a differential operator of order $k$, not necessarily linear, satisfying $D(0)=0$, where $\Gamma(E)$ (resp. $\Gamma(F)$ ) denotes the linear space of all $C^{\infty}$-differentiable cross sections of $E$ (resp. $F$ ). Let $J_{k}(E)$ denote the jet bundle of order $k$ of $E$. There is a unique fiber preserving map $\varphi: J_{k}(E) \rightarrow F$ such that $D(s)=\varphi \circ j^{k}(s)$. We define $p^{1}(\varphi): J_{k+1}(E) \rightarrow$ $J_{1}(F)$, the first prolongation of $\varphi$, by $p^{1}(\varphi)\left(j^{k+1}(s)\right)=j^{1}\left(\varphi\left(j^{k}(s)\right)\right)$. For $\sigma \in \Gamma(F)$, let $A=\varphi^{-1}(\sigma)$ and $A^{(1)}=p^{1}(\varphi)^{-1}\left(j^{1}(\sigma)\right) . A^{(1)}$ is called the first prolongation of $A$. Let $\pi: J_{k+1}(E) \rightarrow M$ and $\pi_{k}: J_{k+1}(E) \rightarrow J_{k}(E)$ be natural projections.

We denote by $T^{*}=T^{*} M$ the cotangent bundle of $M$ and by $S^{k} T^{*}$ the $k$-tuple symmetric product of $T^{*}$. There is a natural vector bundle morphism $i: S^{k} T^{*} \otimes E \rightarrow J_{k}(E)$ and the sequence

$$
0 \longrightarrow S^{k} T^{*} \otimes E \stackrel{i}{\longrightarrow} J_{k}(E) \stackrel{\pi_{k-1}}{\longrightarrow} J_{k-1}(E) \longrightarrow 0
$$

is exact. (cf. [7]) Let $\pi^{*}\left(S^{k} T^{*} \otimes E\right)$ be the vector bundle over $J_{k}(E)$ induced by $\pi$. We define $i_{*}: \pi^{*}\left(S^{k} T^{*} \otimes E\right) \rightarrow T\left(J_{k}(E)\right)$ as follows; for $(p, \alpha) \in \pi^{*}\left(S^{k} T^{*} \otimes E\right)$ where $p \in J_{k}(E)$ and $\alpha \in S^{k} T^{*} \otimes E$,

$$
\left.i_{*}(p, \alpha) \stackrel{\text { def. }}{=} \frac{d}{d t}(p+i(t \alpha))\right|_{t=0}
$$

We have the exact sequence;

$$
0 \longrightarrow \pi^{*}\left(S^{k} T^{*} \otimes E\right) \stackrel{i *}{\longrightarrow} T\left(J_{k}(E)\right) \stackrel{\left(\pi_{k-1}\right) *}{\longrightarrow}\left(\pi_{k-1}\right)^{*} T\left(J_{k-1}(E)\right) \longrightarrow 0 \text {. }
$$

Let $F(A)=\left\{v \in T(A) \mid\left(\pi_{k-1}\right)_{*} v=0\right\}$ and $g=i_{*}^{-1}(F(A)) . \quad g$ is a family of vector spaces over $A$ and is called the symbol of $A$.

Definition 1. The differential equation $D(s)=\sigma$ is said to be involutive at $p \in A$ if there is a neighborhood $U$ of $p$ in $J_{k}(E)$ which satisfies the following two conditions; 
(1) the rank of $\varphi$ is constant on $U$,

(2) let $\tilde{A}=A \cap U$ and $\tilde{A}^{(1)}=\left\{q \in A^{(1)} \mid \pi_{k} q \in U\right\}$, then $\tilde{A}$ is an involutive differential equation in the sense of [2], that is, $\widetilde{A}$ (resp. $\tilde{A}^{(1)}$ ) is a fibered submanifold of $J_{h}(E)$ (resp. $J_{k+1}(E)$ ) and $\pi_{k}: \tilde{A}^{(1)} \rightarrow \tilde{A}$ is a fibered manifold and moreover $\left.g\right|_{\tilde{A}}$ is involutive.

Further, if the equation $D(s)=\sigma$ is involutive at any $p \in A$, then we simply say that $D(s)=\sigma$ is involutive.

Note that in this definition, $\pi_{k}: \widetilde{A}^{(1)} \rightarrow \widetilde{A}$ is actually an affine bundle, and that its associated vector bundle is nothing but the first prolongation $g^{(1)}$ of $g$. See [2] for the details.

Let $D: \Gamma(E) \rightarrow \Gamma(F)$ be as above. The linearization $L$ of $D$ at 0 is defined by $L(s)=\left.\frac{d}{d t} D(t s)\right|_{t=0}$ for $s \in \Gamma(E) . \quad L: \Gamma(E) \rightarrow \Gamma(F)$ is a linear differential operator of order $k$. Let $\varphi_{*}: J_{h}(E) \rightarrow F$ be the differential of $\varphi$ along $j^{k}(0)$, that is, $\varphi_{*}(p)=\left.\frac{d}{d t} \varphi(t p)\right|_{t=0}$. Obviously we have $L=$ $\varphi_{* * j} j^{k}$.

Proposition 1. If $D(s)=0$ is involutive at $j^{k}(0)$, the linear equation $L s=0$ is also involutive.

Proof. Let $U$ be a neighborhood of $j^{k}(0)$ in Definition 1 and $\tilde{A}=$ $A \cap U$, where $A=\varphi^{-1}(0)$. Let $R=\varphi_{*}^{-1}(0)$ and $R^{(1)}=p^{1}\left(\varphi_{*}\right)^{-1}\left(j^{1}(0)\right)$. Remark that $p \in J_{k}(E)_{x}$ can be naturally considered as an element of $T_{j_{x}(0)}^{k} J_{k}(E)$. Under this identification, $p \in J_{k}(E)$ is contained in $R$ if and only if $p$ is tangent to $\tilde{A}$. Let $p^{1}(\varphi)_{*}$ be the differential of $p^{1}(\varphi)$ along $j^{k+1}(0)$. It is easy to see that $p^{1}(\varphi)_{* *}=p^{1}\left(\varphi_{*}\right)$. Let $W$ be a small neighborhood of $x \in M$ and $\left.J_{1}(F)\right|_{W} \cong W \times \mathbb{R}^{l}$ a local trivialization of $J_{1}(F)$. Let $p^{1}(\varphi)=\left(\psi_{1}, \ldots, \psi_{l}\right)$ be the coordinates of $p^{1}(\varphi)$ in this trivialization. Although the rank of $p^{1}(\varphi)$ is not necessarily constant, we can choose functions $\psi_{i_{1}}, \ldots, \psi_{i_{l^{\prime}}}$ such that $\psi_{i_{1}}=\cdots=\psi_{i_{l^{\prime}}}=0$ are regular defining equations of $\tilde{A}^{(1)}$ in a neighborhood of $j_{x}^{k+1}(0)$ and the others are written in the form $\psi_{j}=\sum_{k=1}^{l^{\prime}} f_{i_{k}} \psi_{i_{k}}$, where $f_{i_{k}}$ is a function defined in a neighborhood of $j_{x}^{k+1}(0)$. (To show this, recall that the first prolongation $g^{(1)}$ of $g$ is the vector bundle associated with the affine bundle $\tilde{A}^{(1)} \rightarrow \tilde{A}$, and express this fact by the coordinates of $\varphi$ and their derivatives.) Therefore $p \in J_{k+1}(E)$ is an element of $R^{(1)}$ if and only if $p$ is tangent to $\widetilde{A}^{(1)}$. Since $\pi_{k}: \tilde{A}^{(1)} \rightarrow \tilde{A}$ is a fibered manifold, $R^{(1)}$ and $R$ are vector bundles 
and $\pi_{k}: R^{(1)} \rightarrow R$ is surjective. Moreover we can naturally identify the symbol of $R$ with that of $A$ on $j^{k}(0)$, so that $R$ is involutive. Q.E.D.

In general, let $L: \Gamma(E) \rightarrow \Gamma(F)$ be a linear differential operator of order $k$ such that $L=\varphi \circ j^{k}$. For $\sigma \in \Gamma(F)$, we put $R(\sigma)=\varphi^{-1}(\sigma)$ and $R(\sigma)^{(1)}=p^{1}(\varphi)^{-1}\left(j^{1}(\sigma)\right)$.

Proposition 2. If the equation $L(s)=0$ is involutive, the inhomogeneous equation $L(s)=\sigma$ is involutive if and only if $\pi: R(\sigma)^{(1)} \rightarrow M$ is surjective.

Proof. The "only if" part is trivial. Assume that $\pi: R(\sigma)^{(1)} \rightarrow M$ is surjective. Then $\pi: R(\sigma)^{(1)} \rightarrow M$ is an affine bundle whose associated vector bundle is $R(0)^{(1)}$. From the commutative diagram

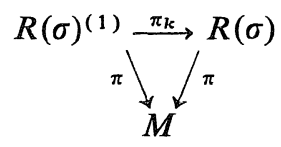

it follows that $\pi: R(\sigma) \rightarrow M$ is also surjective, so $\pi: R(\sigma) \rightarrow M$ is an affine bundle whose associated vector bundle is $R(0)$. Note $\pi_{k}: R(0)^{(1)} \rightarrow R(0)$ is surjective by the assumption, so that $\pi_{k}: R(\sigma)^{(1)} \rightarrow R(\sigma)$ is also surjective affine bundle map. Since the symbol of $R(\sigma)$ is the same as that of $R(0)$, it follows that $R(\sigma)$ is involutive.

Q.E.D.

Let $\Theta$ be the sheaf of germs of all $C^{\infty}$-differentiable solutions of the equation $L(s)=0$ and $\sigma$ a real analytic cross section of $F$.

Proposition 3. If the equation $L(s)=\sigma$ is involutive and $H^{1}(M$, $\Theta)=0$, there exists a global solution of the equation $L(s)=\sigma$.

Proof. By Cartan-Kähler theorem, there is a covering $\mathscr{U}=\left\{U_{\alpha}\right\}$ of $M$ and cross sections $s_{\alpha}$ over $U_{\alpha}$ such that $L\left(s_{\alpha}\right)=\sigma$. We have $L\left(s_{\alpha}-\right.$ $\left.s_{\beta}\right)=0$ on $U_{\alpha} \cap U_{\beta}$, and hence the assignment

$$
(\alpha, \beta) \longrightarrow s_{\alpha}-s_{\beta}
$$

is a 1-cochain of $\Theta$. It is easily seen that this is a cocycle. Since $H^{1}(\mathscr{U}, \Theta) \rightarrow H^{1}(M, \Theta)$ is injective, we have $H^{1}(\mathscr{U}, \Theta)=0$. Therefore 
there are cross sections $s_{\alpha}^{\prime}$ of $\Theta$ over $U_{\alpha}$ such that $s_{\alpha}-s_{\beta}=s_{\beta}^{\prime}-s_{a}^{\prime}$. Then $s_{0}=s_{\alpha}+s_{\alpha}^{\prime}$ is a global solution of the equation $L(s)=\sigma$.

q.e.d.

Finally we mention a class of differential operators. Let $D$ be a non-linear operator such that $D=\varphi \circ j^{k}$. Define $\varphi^{\mu} \in S^{\mu}\left(J_{k}(E)^{*}\right) \otimes F$ by

$$
\varphi^{\mu}\left(p_{1}, \ldots, p_{\mu}\right)=\left.\frac{1}{\mu !} \frac{\partial^{\mu}}{\partial t_{1} \ldots \partial t_{\mu}} \varphi\left(t_{1} p_{1}+\cdots+t_{\mu} p_{\mu}\right)\right|_{t_{1}=\cdots=t_{\mu}=0} .
$$

For $\sigma \in \Gamma\left(J_{k}(E)\right), \varphi(\sigma)=\sum_{\mu=0}^{\infty} \varphi^{\mu}(\sigma, \ldots, \sigma)$ is the formal Taylor expansion of $\varphi$ at $j^{k}(0)$. The right hand converges in each fiber if $\sigma$ is sufficiently close to $j^{k}(0)$. Define $D^{\mu}: \Gamma(E) \times \cdots \times \Gamma(E) \rightarrow \Gamma(F)$ by $D^{\mu}\left(s_{1}, \ldots, s_{\mu}\right)=$ $\varphi^{\mu}\left(j^{k}\left(s_{1}\right), \ldots, j^{k}\left(s_{\mu}\right)\right)$.

Definition 2. $D$ is called a polynomial differential opcrator of degree $n$ if $D^{\mu}=0$ for $\mu>n$.

\section{§2. The Construction of Deformations}

As in the beginning of $\S 1$, let $D: \Gamma(E) \rightarrow \Gamma(F)$ be a non-linear operator of order $k$ such that $D(0)=0$ and $D=\varphi \circ j^{k}$. Let $L$ be the linearization of $D$ at 0 and $\Theta$ the solution sheaf of the equation $L(s)=0$.

Let $s(t)$ be a parametrized family of cross sections of $E$, where $t$ moves in some neighborhood of 0 in a euclidean space and $s(t)$ depends real analytically on $t$. We say that $s(t)$ is a deformation of the solution 0 if $s(0)=0$ and $D(s(t))=0$.

We can prove

Theorem 1. Assume the followings:

(1) $D$ is a polynomial operator of degree $n$,

(2) the equation $D(s)=0$ is involutive at $j^{k}(0)$,

(3) $L$ is elliptic and $H^{1}(M, \Theta)=0$.

Then there exists a deformation $s(t)$ such that

(4) 1 moves in a neighborhood of 0 in $\mathbf{R}^{m}$, where $m=\operatorname{dim} H^{0}(M$, $\Theta)$,

(5) the linear mapping

$$
T_{0} \mathbb{R}^{m} \ni \Sigma \lambda_{l} \partial / \partial t_{i} \longrightarrow \Sigma \lambda_{i}\left(\frac{\partial s(t)}{\partial t_{i}}\right)_{t-0} \in H^{0}(M, \Theta)
$$


is bijective.

Proof. The proof will be divided into two steps.

a. (Existence of formal solutions) We want to construct homogeneous polynomials $s_{r}(t)$ of $t_{1}, \ldots, t_{m}$ of degree $r$ with coefficients in $\Gamma(E)$ such that $u_{r}(t)=s_{1}(t)+s_{2}(t)+\cdots+s_{r}(t)$ satisfies

$$
D\left(u_{r}(t)\right) \equiv 0 \quad \bmod t^{r+1}
$$

where we mean, for any polynomial $\psi(t)$ of $t_{1}, \ldots, t_{m}$, by $\psi(t) \equiv 0 \bmod t^{r+1}$ that $\psi(t)$ contains no terms of degree $\leqq r$.

Let $\zeta_{1}, \ldots, \xi_{m}$ be a basis of the vector space $H^{0}(M, \Theta)$ and set

$$
s_{1}(t)=\xi_{1} t_{1}+\check{\zeta}_{2} t_{2}+\cdots+\ddot{\zeta}_{m} t_{m}
$$

It is obvious that $u_{1}(t)=s_{1}(t)$ satisfies $(2.1)_{1}$. It should be noticed that each $\xi_{i}$ is an analytic cross section. Suppose that $s_{1}(t), \ldots, s_{r}(t)$ are already determined in such a way that the coefficients of $s_{i}(t)$ are analytic cross sections. Then we have to construct $s_{r+1}(t)$ satisfying

$$
D\left(u_{r}(t)+s_{r+1}(t)\right) \equiv 0 \quad \bmod t^{r+2} \text {. }
$$

Let $D^{\mu}=\varphi^{\mu} \circ j^{k}$ be the operator dcfined in $\S 1$. Clearly we have $D^{1}=L$ and $D^{0}=D(0)=0$. Let $\eta_{r+1}(t)$ be the homogeneous element of degree $r+1$ of $D\left(u_{r}(t)\right)$. We get

$$
\begin{aligned}
& D\left(u_{r}(t)+s_{r+1}(t)\right)=\sum_{\mu=1}^{n} D^{\mu}\left(u_{r}(t)+s_{r+1}(t), \ldots, u_{r}(t)+s_{r+1}(t)\right) \\
& \equiv L\left(s_{r+1}(t)\right)+\sum_{\mu=1}^{n} D^{\mu}\left(u_{r}(t), \ldots, u_{r}(t)\right) \quad \bmod t^{r+2} .
\end{aligned}
$$

Hence (2.2) is equivalent to

$$
L\left(s_{r+1}(t)\right)+\eta_{r+1}(t)=0 .
$$

We will show that the differential equation (2.3) is involutive. Let $p(t)$ be a homogeneous polynomial in $t_{1}, \ldots, t_{m}$ of degree $r+1$ with coefficients in the vector space $J_{k}(E)_{x}$ over $x \in M$. The formal version of (2.3) is

$$
\varphi_{*}(p(t))+\eta_{r+1}(t)=0
$$


or

$$
\varphi\left(p(t)+j_{x}^{k}\left(u_{r}(t)\right)\right) \equiv 0 \quad \bmod t^{r+2} .
$$

Let $U$ be a sufficiently small neighborhood of $x$ and $\rho^{-1}(U) \cong U \times \mathbb{R}^{l}$ a local trivialization of $F$. Let $\varphi=\left(\varphi_{1}, \ldots, \varphi_{l}\right)$ be the coordinates of $\varphi$ in this trivialization. Since the rank of $\varphi$ is constant, we may assume that $\varphi_{1}, \ldots, \varphi_{l^{\prime}}$ is independent in a neighborhood of $j_{x}^{k}(0)$. Hence there are functions $\Psi_{a}$ such that $\varphi_{a}(p)=\Psi_{a}\left(\varphi_{1}(p), \ldots, \varphi_{l^{\prime}}(p)\right)$ for $l^{\prime}<a \leqq l$ if $p$ is sufficiently close to $j_{x}^{k}(0)$. Let $\tilde{\varphi}=\left(\varphi_{1}, \ldots, \varphi_{l^{\prime}}\right)$, which is considered as a map from $\left.J_{k}(E)\right|_{U}$ into a subbundle of $\left.F\right|_{U}$. Since $\tilde{\varphi}_{*}$ is surjective, there is a homogencous polynomial $p(t)$ such that

$$
\tilde{\varphi}\left(p(t)+j_{\lambda}^{h}\left(u_{r}(t)\right)\right) \equiv 0 \quad \bmod t^{r+2} .
$$

Then it follows from the above arguments that (2.4) holds for this $p(t)$. Although the rank of $p^{1}(\varphi)$ is not constant, by the arguments in the proof of Proposition 1, we can similarly show that there is a homogeneous polynomial $p^{\prime}(t)$ of degree $r+1$ with coefficients in $J_{k+1}(E)_{x}$ such that

$$
p^{1}(\varphi)\left(p^{\prime}(t)+j_{x}^{h+1}\left(u_{r}(t)\right)\right) \equiv 0 \quad \bmod t^{r+2} .
$$

Remark that (2.5) is equivalent to

$$
p^{1}\left(\varphi_{*}\right)\left(p^{\prime}(t)\right)+j_{x}^{1}\left(\eta_{r+1}(t)\right)=0
$$

Since the equation $L(s)=0$ is involutive, in view of Proposition 2, it follows that $(2.3)$ is involutive.

Finally by Proposition 3, there is a global solution of (2.4) and further the ellipticity of $L$ implies that it is analytic. This completes our inductive construction of $s(t)$.

b. (Proof of convergence) We introduce the norm $\|s\|_{p+\sigma}$ of $s \in$ $\Gamma(E)$ (or $s \in \Gamma(F)$ ) for a positive integer $p$ and $0<\sigma<1$ by a well-known method. That is, let $\left\{U_{i}\right\}$ be a finite covering of $M$ and $\left\{x_{1}, \ldots, x_{n}\right\}$ a coordinate system on $U_{i}$. (Recall that $M$ is compact) We write $s$ in the form $s=\Sigma s_{\lambda} e_{\lambda}$, where $\left\{e_{\lambda}\right\}$ is a set of linearly independent local cross sections of $E$ (or $F$ ). Let 


$$
\begin{aligned}
\|s\|_{p+\sigma} & =\sup _{i}\|s\|_{p+\sigma}^{U_{i}} \\
\|s\|_{p+\sigma}^{U_{i}} & =\sum_{r=0}^{p} \sup \left|\partial^{r} s_{\lambda}(x)\right|+\sup \frac{\left|\partial^{p} s_{\lambda}(x)-\partial^{p} s_{\lambda}(y)\right|}{|x-y|^{\sigma}}
\end{aligned}
$$

where $\partial^{r}$ means a partial derivative of order $r$ and the "sup" is extended over all points $x, y \in U_{i}$, all indices $\lambda$ and all partial derivatives $\partial^{r}, \partial^{p}$.

For $p>k$, we have

$$
\left\|D^{\mu}\left(s_{1}, \ldots, s_{\mu}\right)\right\|_{p-k+\sigma} \leqq c\left\|s_{1}\right\|_{p+\sigma} \ldots\left\|s_{\mu}\right\|_{p+\sigma}
$$

where $k$ is the order of $D$.

Let $s$ be a global solution of the equation $L(s)=s^{\prime}$ for $s^{\prime} \in \Gamma(F)$. It is known that the ellipticity of $L$ implies the estimate (cf. [1])

$$
\|s\|_{p+\sigma} \leqq c\left(\|s\|_{0}+\left\|s^{\prime}\right\|_{p-k+\sigma}\right) .
$$

Moreover by the arguments similar to section 4 of [3], it follows that there exists a solution $s^{\prime \prime}$ such that the next estimate holds:

$$
\left\|s^{\prime \prime}\right\|_{p+\sigma} \leqq c_{1}\left\|s^{\prime}\right\|_{p-k+\sigma}
$$

where $c_{1}$ is independent of $s^{\prime}$.

Let $\alpha$ denote a multi-index $\left(\alpha_{1}, \ldots, \alpha_{m}\right)$ and consider a formal power series

$$
s(t)=\Sigma s_{\alpha} t^{\alpha}=\Sigma s_{\alpha_{1} \cdots \alpha_{m}} t_{1}^{\alpha_{1}} \ldots t_{m}^{\alpha_{m}}
$$

with coefficients $s_{\alpha} \in \Gamma(E)$ (or $\in \Gamma(F)$ ) and a power series $a(t)=\Sigma a_{\alpha} t^{\alpha}$. We indicate by $\|s\|_{p+\sigma}(t) \ll a(t)$ that $\left\|s_{\alpha}\right\|_{p+\sigma} \leqq a_{\alpha}$.

We will make use of the power series introduced in [3]. That is, let

$$
A(t)=\frac{a}{64 b} \sum_{r=1}^{\infty} \frac{b^{r}\left(t_{1}+\cdots+t_{m}\right)^{r}}{r^{2}}
$$

We have

$$
A(t)^{r} \ll\left(\frac{a}{b}\right)^{r-1} A(t)
$$

Now we proceed to the proof of convergence. First remark that 
from (2.8), there is a global solution $s_{r+1}(t)$ of (2.3) such that

$$
\left\|s_{r+1}\right\|_{p+\sigma}(t) \ll c_{1}\left\|\eta_{r+1}\right\|_{p-k+\sigma}(t) .
$$

Let $s(t)=s_{1}(t)+s_{2}(t)+s_{3}(t)+\cdots$, where each $s_{r+1}(t)$ is the solution of (2.3) satisfying (2.10). We want to show

$$
\|s\|_{p+\sigma}(t) \ll A(t) .
$$

By letting a sufficiently large, we may suppose $\left\|u_{1}\right\|_{p+\sigma}(t) \ll A(t)$. We will show (2.11) by induction. Assume

$$
\left\|u_{r}\right\|_{p+\sigma}(t) \ll A(t) .
$$

Since $D\left(u_{r}(t)\right)=\sum_{\mu=1}^{n} D^{\mu}\left(u_{r}(t), \ldots, u_{r}(t)\right)$ and $\eta_{r+1}(t)$ is the homogeneous part of degree $r+1$ of $D\left(u_{r}(t)\right)$, we get from (2.6), (2.9) and (2.12)

$$
\begin{aligned}
\left\|\eta_{r+1}\right\|_{p-k+\sigma}(t) & \ll c \sum_{\mu=2}^{n} A(t)^{\mu} \\
& \ll c \sum_{\mu=2}^{n}\left(\frac{a}{b}\right)^{\mu-1} A(t) .
\end{aligned}
$$

Choosing $b$ so that $c c_{1} \sum_{\mu=1}^{n-1}\left(\frac{a}{b}\right)^{\mu}<1$, we get finally from (2.10)

$$
\left\|s_{r+1}\right\|_{p+\sigma}(t) \ll A(t) .
$$

This completes the proof of (2.11).

Therefore the series

$$
s(t)=s_{1}(t)+s_{2}(t)+s_{3}(t)+\cdots
$$

converges in \|\|$_{p+\sigma}$ for sufficiently small $t$, so that $s(t)$ is differentiable of class $C^{p}$ and real analytic in $t$. Finally we must prove that $s(t)$ is real analytic. This can be shown as follows; in the first place, since $J_{k}(E)$ is naturally imbedded in $J_{1}\left(J_{k-1}(E)\right)$, we can find a certain first order differential operator $D^{\prime}$ on $J_{k-1}(E)$ such that the equation $D(s)=0$ is equivalent to the equation $D^{\prime}\left(j^{k-1}(s)\right)=0$. That is, for a local cross section $s$ of $E, D(s)=0$ if and only if $D^{\prime}\left(j^{k-1}(s)\right)=0$. ( $D^{\prime}$ may be defined only locally, but it suffices for our purpose.) We assert that the linearization of $D^{\prime}$ at $j^{k-1}(0)$ is elliptic. In fact, this is easily seen by direct 
calculations in local coordinate systems. Let us complexify the bundle $E$ and the operator $D^{\prime}$. We can suppose that $s(t)$ is defined for complex numbers $t$. Then $s(t)$ is a solution of the differential equation

$$
\left\{\begin{array}{l}
D^{\prime}\left(j^{k-1}(s(t))\right)=0 \\
\frac{\partial j^{k-1}(s(t))}{\partial \tilde{t}_{i}}=0
\end{array}\right.
$$

The linearization of this equation at $j^{k-1}(s(t))$ is elliptic if $t$ is sufficiently close to 0 , and hence we see that $s(t)$ is real analytic. (cf. [6]) This completely proves the theorem.

Q.E.D.

\section{§3. The Completeness of $s(t)$ at $t=0$}

The notations being the same as in $\$ 2$, in this section we show the completeness of $s(t)$ at $t=0$.

Let $s(t)$ be an arbitrary deformation, where $t$ moves in a neighborhood of 0 in $\mathbf{R}^{m}$.

Definition 3. $s(t)$ is said to be complete at $t=0$ if for any deformation $s^{\prime}(u)$, where $u$ moves in a neighborhood of 0 in $\mathbb{R}^{n}$, there exists a real analytic map $f$ from a neighborhood $V$ of 0 in $\mathbb{R}^{n}$ into $\mathbb{R}^{m}$ such that $f(0)=0$ and $s^{\prime}(u)=s(f(u))$ for $u \in V$.

In the next theorem, we do not assume that $D$ is a polynomial operator.

Theorem 2. Let $s(t)$ be a deformation such that the natural mapping

$$
T_{0} \mathbf{R}^{m} \ni \Sigma \lambda_{i} \partial / \partial t_{i} \longrightarrow \Sigma \lambda_{i}\left(\frac{\partial s(t)}{\partial t_{i}}\right)_{t=0} \in H^{0}(M, \Theta)
$$

is surjective. Then $s(t)$ is complete at $t=0$.

Proof. As the proof of Theorem 1, we first construct $f$ formally, next prove its convergence.

a. Let $s^{\prime}(u)$ be any deformation. We want to construct $f$ such that 


$$
s^{\prime}(u) \equiv s(f(u)) \quad \bmod u^{r+1}
$$

for any $r$. Let $s(t)=\sum_{\alpha} s_{\alpha} t^{\alpha}$ and $s^{\prime}(u)=\sum_{\beta} s_{\beta}^{\prime} u^{\beta}$ be the Taylor expansions of $s(t)$ and $s^{\prime}(u)$. We put $s_{r}(t)=\sum_{|\alpha|=r} s_{\alpha} t^{\alpha}$ and $s_{r}^{\prime}(u)=\sum_{|\beta|=r} s_{\beta}^{\prime} u^{\beta}$, where $|\alpha|=\alpha_{1}+\alpha_{2}+\cdots+\alpha_{m}$ and $|\beta|=\beta_{1}+\beta_{2}+\cdots+\beta_{n}$. Similarly we write $f$ in the form $f(u)=\sum_{\beta} f_{\beta} u^{\beta}=\sum_{r=1}^{\infty} f_{r}(u)$, which we must construct such that (3.1) holds. Let $f_{\beta}=\left(f_{\beta}^{1}, \ldots, f_{\beta}^{m}\right)$ be the coordinates of $f_{\beta}$. (3.1)r is clearly equivalent to

$$
\sum_{i=1}^{r} s_{i}^{\prime}(u) \equiv \sum_{i=1}^{r} s_{i}\left(\sum_{j=1}^{r} f_{j}(u)\right) \quad \bmod u^{r+1}
$$

We denote by $1_{i}$ the multi-index whose $i$-component is 1 and others are 0. Then (3.2) means that $s_{1_{i}}^{\prime}=\sum_{j=1}^{n} s_{1_{j}} f_{1_{i}}^{j}$. Since $s_{1_{i}}^{\prime}$ is an element of $H^{0}(M, \Theta)$ and $\left\{s_{1_{j}}\right\}_{j=1}^{m}$ generate $H^{0}(M, \Theta)$, we can find $f_{1}^{j}$, which satisfy this equation.

Suppose that $f_{1}(u), \ldots, f_{r}(u)$ are already constructed. We must determine $f_{r+1}(u)$ which satisfies (3.2) $)_{r+1}$. Substituting $\sum_{i=1}^{r} f_{i}(u)$ for $t$, we develop $t^{\alpha}$ in $u$ as follows;

$$
t^{\alpha}=\left(\sum_{i=1}^{r} f_{i}(u)\right)^{\alpha}=\sum_{\beta} \sigma_{\beta}^{\alpha} u^{\beta}
$$

Obviously $\sigma_{\beta}^{\alpha}=0$ if $|\beta|<|\alpha|$ or if $|\alpha|=1$ and $|\beta|=r+1$. It is easy to see that $(3.2)_{r+1}$ is equivalent to

$$
\sum_{|\beta|=r+1} s_{\beta}^{\prime} u^{\beta}=\sum_{|\beta|=r+1} s_{1_{i}} f_{\beta}^{i} u^{\beta}+\sum_{|\beta|=r+1} \sum_{\alpha} s_{\alpha} \sigma_{\beta}^{\alpha} u^{\beta}
$$

or

$$
s_{\beta}^{\prime}-\sum_{\alpha} s_{\alpha} \sigma_{\beta}^{\alpha}=\sum_{i} s_{1_{i}} f_{\beta}^{i},|\beta|=r+1
$$

We now prove that the left hand in (3.3) is contained in $H^{0}(M, \Theta)$. In the first place, we get from (3.2)r

$$
s_{\beta}^{\prime}=\sum_{\alpha} s_{\alpha} \sigma_{\beta}^{\alpha}
$$

for $|\beta| \leqq r$. Let $\left\{\alpha_{1}, \ldots, \alpha_{\mu}\right\}$ be multi-indices and set $\alpha=\alpha_{1}+\cdots+\alpha_{\mu}$. Since $t^{\alpha}=t^{\alpha_{1}} \ldots t^{\alpha_{\mu}}$, it is easily shown that 


$$
\sigma_{\beta}^{\alpha}=\sum_{\beta_{1}+\ldots+\beta_{\mu}=\beta} \sigma_{\beta_{1}}^{\alpha} \ldots \sigma_{\beta_{\mu}}^{\alpha_{\mu}}
$$

We remark that a similar equation to (2.3) holds for $s(t)$ and $s^{\prime}(u)$. Since $\eta_{r+1}(t)$ in (2.3) is the homogeneous element of degree $r+1$ of

$$
\begin{aligned}
D\left(\sum_{i=1}^{r} s_{i}(t)\right) & =\sum_{\mu \geqq 1} D^{\mu}\left(\sum_{i=1}^{r} s_{i}(t), \ldots, \sum_{i=1}^{r} s_{i}(t)\right) \\
& =\sum_{\alpha, \mu} \sum_{\substack{\alpha_{1}+\ldots+\alpha_{\mu}=\alpha \\
\left|\alpha_{i}\right| \leqq}} D^{\mu}\left(s_{\alpha_{1}}, \ldots, s_{\alpha_{\mu}}\right) t^{\alpha}
\end{aligned}
$$

it follows that

$$
L\left(s_{\alpha}\right)=-\sum_{\mu \geqq 2} \sum_{\alpha_{1}+\cdots+\alpha_{\mu}=\alpha} D^{\mu}\left(s_{\alpha_{1}}, \ldots, s_{\alpha_{11}}\right)
$$

for $|\alpha|=r+1$. Similarly we have

$$
L\left(s_{\beta}^{\prime}\right)=-\sum_{\mu \geqq 2} \sum_{\beta_{1}+\cdots+\beta_{\mu}=\beta} D^{\mu}\left(s_{\beta_{1}}^{\prime}, \ldots, s_{\beta_{\mu}}^{\prime}\right) .
$$

Let us return to the equation (3.3). We get from $(3.4) \sim(3.7)$

$$
\begin{aligned}
L\left(s_{\beta}^{\prime}\right) & =-\sum_{\mu \geqq 2} \sum_{\beta_{1}+\ldots+\beta_{\mu}=\beta} D^{\mu}\left(\sum_{\alpha_{1}} s_{\alpha_{1}} \sigma_{\beta}^{\alpha}, \ldots, \sum_{\alpha, \mu} s_{\alpha_{\mu}} \sigma_{\beta_{\mu}}^{\alpha_{\mu}}\right) \\
& =-\sum_{\mu \geqq 2, \alpha} \sum_{\alpha_{1}+\cdots+\alpha_{\mu}=\alpha} D^{\mu}\left(s_{\alpha_{1}}, \ldots, s_{\alpha_{\mu}}\right) \sigma_{\beta}^{\alpha} \\
& =\sum_{\alpha} L\left(s_{\alpha}\right) \sigma_{\beta}^{\alpha} .
\end{aligned}
$$

This shows that $L\left(s_{\beta}^{\prime}-\sum_{\alpha} s_{\alpha} \sigma_{\beta}^{\alpha}\right)=0$. Hence we can find $f_{\alpha}^{i}$ which satisfy (3.3). This completes our inductive construction of $f(u)$.

Note that we can choose a basis of $H^{0}(M, \Theta)$ between $\left\{s_{1_{i}}\right\}_{i=1}^{m}$. For simplicity, let $\left\{s_{1_{i}}\right\}_{i=1}^{m^{\prime}}$ be a basis. Then we can suppose in the construction of $f_{\alpha}^{i}$ that $f_{\alpha}^{i}=0$ for $i>m^{\prime}$.

b. Let $<,>$ be a metric in $E$ and define an inner product of $\Gamma(E)$ by

$$
\left(s, s^{\prime}\right)=\int_{M}<s(x), s^{\prime}(x)>d v
$$

where $d v$ is a volume element of $M$. Let $\|s\|=(s, s)^{\frac{1}{2}}$ be the norm of 
$s \in \Gamma(E)$. Since the inner product restricted to $H^{0}(M, \Theta)$ is positive definite and $\left\{s_{1_{i}}\right\}_{i=1}^{m^{\prime}}$ is a basis of $H^{0}(M, \Theta)$, there is a constant $c$ such that

$$
\left|f_{\beta}^{i}\right| \leqq c\left\|\sum_{k=1}^{m} s_{1_{k}} f_{\beta}^{k}\right\|
$$

(Remark that $f_{\alpha}^{k}=0$ for $k>m^{\prime}$ ) Further, by Cauchy's inequality and simple calculations, we can suppose that

$$
\begin{gathered}
\|s\|(t) \ll A_{0}(t) \\
\left\|s^{\prime}\right\|(u) \ll A_{0}(u)
\end{gathered}
$$

where

$$
\begin{aligned}
A_{0}(t) & =\frac{a_{0}}{64 b_{0}} \sum_{r=1}^{\infty} \frac{b_{0}^{r}\left(t_{1}+\cdots+t_{m}\right)^{r}}{r^{2}} \\
A_{0}(u) & =\frac{a_{0}}{64 b_{0}} \sum_{r=1}^{\infty} \frac{b_{0}^{r}\left(u_{1}+\cdots+u_{n}\right)^{r}}{r^{2}} .
\end{aligned}
$$

Let $A(u)$ be the power series $A_{0}(u)$ in which the constant $a_{0}, b_{0}$ are replaced by $a, b$ and assume that $\sum_{r=1}^{i} f_{i}^{k}(u) \ll A(u)$ for any $k$. We have

$$
\sum_{\beta} \sigma_{\beta}^{\alpha} u^{\beta}=\left(\sum_{i=1}^{r} f_{i}(u)\right)^{\alpha} \ll A(u)^{|\alpha|} \ll\left(\frac{a}{b}\right)^{|\alpha|-1} A(u) .
$$

Let $A_{\alpha}$ denote the coefficient of $t^{\alpha}$ in $A_{0}(t)$. Then we get from (3.3),

$$
\sum_{|\beta|=r+1}\left|f_{\beta}^{i}\right| u^{\beta} \ll c\left\{A_{0}(u)+\sum_{r+1 \geqq|\alpha| \geqq 2} A_{\alpha}\left(\frac{a}{b}\right)^{|\alpha|-1} A(u)\right\}
$$

We may suppose that $a_{0}<a, b_{0}<b$, and hence we can replace $A_{0}(u)$ by $\frac{b_{0}}{b} A(u)$ in (3.10). It is easily shown that we can choose $b$ such that

$$
c\left(\frac{b_{0}}{b}+\sum_{|\alpha| \geqq 2} A_{\alpha}\left(\frac{a}{b}\right)^{|\alpha|-1}\right)<1 .
$$

This shows that $\sum_{i=1}^{r+1} f_{i}^{k}(u) \ll A(u)$, so that we have $f^{k}(u) \ll A(u)$ by induc- 
tion. Therefore $f(u)$ converges in a sufficiently small neighborhood of 0 and we complete the proof. $\quad$ Q.E.D.

\section{References}

[1] Douglis, A. and Nirenberg, L., Interior estimates for elliptic systems of partial differential equations, Comm. on Pure and Applied Math., 8 (1955), 503-538.

[2] Goldschmidt, H., Integrability criteria for systems of non-linear partial differential equations, J. Differ. Geom. 1 (1967), 269-307.

[ 3 ] Kodaira, K., Nirenberg, L. and Spencer, D. C., On the existence of deformations of complex analytic structures, Annals of Math., 68 (1958), 450-459.

[4] Kodaira, K. and Spencer, D. C., A theorem of completeness for complex analytic fiber spaces, Acta. Math., 100 (1958), 281-294.

[5] Kuranishi, M., Lectures on exterior differential systesm, Tata Institute of Fundamental Research, Bombay, (1962).

[6] Morrey, C. B., On the analyticity of the solutions of analytic nonlinear elliptic systems of partial differential equations, Amer. J. Math., 80 (1958), 198-237.

[7] Quillen, D. G., Formal properties of over-determined systems of linear partial differential equations, Ph. D. thesis, Harvard University, (1964). 